\title{
COVID-19 Vaccine Comparison: How to Choose the Best Suiting Vaccine for Different Needs
}

\author{
Chuyao Liu ${ }^{1, \mathrm{a},{ }^{*}, \dagger}$, Aiyun $\mathrm{Lu}^{2, \mathrm{~b}, *, \dagger}$, Xiaoyu $\mathrm{Wu}^{3, \mathrm{c}, *, \dagger}$ \\ ${ }^{1}$ School of Chemical and Biomedical Engineering, Nanyang Technological University, Singapore \\ ${ }^{2}$ Department of Biological Sciences, University of Toronto Scarborough, Toronto, Ontario, CA \\ ${ }^{3}$ Department of Biology, Macalester College, Saint Paul, Minnesota, USA \\ $\dagger$ These authors contributed equally.
}

\begin{abstract}
Varied measurements have been developed to solve the COVID-19 pandemic, and one of the best ways is vaccines. This paper aims to compare different kinds of vaccines and help people or countries to choose the best suitable vaccine needs based on their different needs. It provides a broader and more detailed analysis of variant vaccines on different levels. In the sequence of technology maturity, inactivated vaccines including CoronaVac, BBIBP-CorV vaccine, and WIBP-CorV vaccine, viral-based vector vaccines including AstraZeneca COVID-19 vaccine and Johnson \& Johnson's COVID-19 vaccine, and mRNA vaccines including Moderna vaccine and Pfizer/BioNTech vaccine have been discussed. There are several important elements of these vaccines, for example, mechanism, effectiveness, and side effects. Different criteria make comparison. For effectiveness, in general, Moderna and Pfizer/BioNTech vaccines could be a better choice. For cost-performance ratio, the AstraZeneca COVID-19 vaccine is the best. As for inoculators' age and race, old people are suggested to take CoronaVac, and Pfizer/BioNTech vaccine and CoronaVac are suitable for all races. In terms of mutant variant distribution, CoronaVac could be the best choice. Based on safety concerns, inactivated vaccines are better choices. For the developing period, mRNA vaccines win the game. In conclusion, this paper provides further directions for countries seeking the best suitable vaccine for their citizens. After comparing the main vaccines available, we provide a clear view of when countries decide. There would be specific vaccines best suiting specific needs of inoculators and countries.
\end{abstract}

\section{Introduction}

The pandemic of coronavirus disease 2019 is caused by a virus known as the severe acute respiratory syndrome coronavirus 2 (SARS-CoV-2) [1]. Like all coronaviruses, SARS-CoV-2 invades the host cell through binding its spike protein $(180-\mathrm{kDa}$ spike) to the cellular receptor angiotensin-converting enzyme (ACE2) of the host cell and downregulating ACE2 expression (one of the factors for disease progression) [2]. While vaccination is a critical tool for battling against COVID-19, the spike protein is the focus of most COVID-19 vaccines as it is the part of the virus that could be used to trigger the imitation infection during the vaccination, letting our immune systems produce T-lymphocytes and antibodies for fighting against the infection. There are mainly three types of COVID-19 vaccines available for use: inactivated vaccine, viral vector-based vaccine, and mRNA vaccine. Though all COVID-19 vaccines aim to trigger the secondary immune response and neutralize the virus before it makes people sick, they may work through different pathways and have different efficacy and safety profiles. Here come the most difficult questions for countries and people around the world: what types of techniques should be incorporated into vaccine development and what type of vaccine we should choose when they become available? In our paper, we primarily introduce these three kinds of vaccines from the type of vaccine that uses the most classic technique to use the most up-to-date technique, providing information of their mechanisms, efficacies, effectiveness, and safety profiles. Then, we make a comparative analysis among these three types of vaccines. We firstly suggest suitable vaccine techniques for all countries based on concerns on vaccine effectiveness and cost-performance ratio. Then, we suggest suitable vaccines for specific regions based on concerns on age, race, mutant variant distribution, the time needed to develop a new vaccine, and the maturity of the technique used in vaccine development.

\section{General Vaccine Mechanism}

Vaccines containing inactive parts of the antigen or the instructions for producing antigens help develop immunity by imitating an infection without causing illness. The vaccine triggers the immune system to

\footnotetext{
* Corresponding author: ${ }^{\mathrm{B}} \mathrm{B} 180075 @$ e.ntu.edu.sg

baiyun.lu@mail.utoronto.ca

cxwu@macalester.edu.
} 
produce T-lymphocytes and antibodies once injected. Once the imitation infection goes away, the body is left with a supply of "memory" T-lymphocytes and Blymphocytes ready for fighting against the disease in the future infection.

\section{Inactivated Vaccine}

\subsection{Mechanism}

The particular type of vaccine is a modified form of the pathogen whose disease-inducing ability is weakened or removed. When injected inactivated virus vaccines, the immune responses in human bodies are activated. The epitopes of the modified virus can be recognized and remembered; thus, the body is "prepared" for the potential next infection of the same or similar pathogens. Some may require several times of vaccination to induce the function of the immune system fully.

\subsection{Efficacy and Effectiveness}

Effectiveness from $70 \%$ to $90 \%$ is observed in inactivated virus vaccine. For example, the CoronaVac, developed by Sinovac, shown effectiveness at $91.25 \%$ [3]. However, BBIBP-CorV and WIBP-CorV have effectiveness at $79.34 \%$ and $72.51 \%$, respectively $[4,5]$. The data are presented in [3, 4, 5, Fig. 1] CoronaVac is developed by Sinovac, and it has been in Phase III clinical trials in many countries, including Brazil, Chile, Indonesia. The company found good results with inactivated virus-based products (CoronaVac), which is also under normal conditions good for up to 3 years to use. As for CoronaVac, is safe in older adults, and $3 \mu \mathrm{g}$ is the most effective dose examined [6]. The effectiveness was proved to be $91.25 \%$ based on a clinical trial in Brazil [7]. However, it was to some degree doubtful since some Brazilian researchers revealed that the effectiveness was $50.4 \%$. The efficacy against the variants is still unknown. BBIBP-CorV, also known as the Sinopharm COVID-19 vaccine, is one of two inactivated virus COVID-19 vaccines developed by Sinopharm. Same as BBIBP-CorV, The WIBP-CorV vaccine was also developed by a Chinese institute Sinopharm.

\subsection{Side Effects}

In general, no severe side effects were found in people administrated with inactivated virus vaccine. CoronaVac was well tolerated, and no dose-related safety concerns were observed [6]. BBIBP-CorV is well-tolerated at all tested doses in two age groups. (18-59 years and $\geq 60$ years) [7]. Very safe, without the risk of the virus or bacteria mutating back into its disease-causing pathogen, it can be freeze-dried and easily stored [8]. The safety, effectiveness of variants of WIBP-CorV and CoronaVac need to be investigated. of information when

\section{Viral-based Vector Vaccine}

\subsection{Mechanism}

For COVID-19 viral vector vaccines, the vector enters a cell in our body and then uses our cell's machinery to produce a harmless piece of the virus (spike protein) that causes COVID-19. Our immune system recognizes cells displaying the spike protein as an antigen, triggering our immune system to produce antibodies and memory T-cells and B-cells, which will circulate in the blood and help us fight against future disease infection [9].

\subsection{Efficacy and Effectiveness}

The general efficacy of viral vector vaccines is about $70 \%$, with AstraZeneca COVID-19 vaccine (AZN vaccine) at $70.4 \%$ and Johnson \& Johnson's COVID-19 vaccine (J\&J vaccine) at $66 \%[10,11]$. And all viral vector vaccines are more effective at preventing severe disease. For example, the AZN vaccine is $79 \%$ effective in preventing symptomatic illness and $100 \%$ effective against severe disease and hospitalization [12]. Comparisons are shown in $[10,11,12$, Fig. 1]

For efficacies in people over 60, different viral vector vaccines show different results. For example, the J\&J vaccine only has $42.3 \%$ efficacy, while the AZN vaccine has over $80 \%$ efficacy in people older than 60 [13]. However, due to limited data in subsets, we may consider the uncertainty about the accuracy of the efficacy estimate.

For the three major strains of variants we have discussed in the previous section [14], the viral vector vaccines perform similarly against the three variants: both J\&J and AZN vaccines are less effective against the B.1.1.7 variant detected in the UK than against other variants. And they both are less effective against the South Africa variant, whereas being more effective against Brazil variant [15-17].

\subsection{Side Effects}

Although no serious adverse events or deaths were found associated with viral vector vaccination in the clinical trials before, both J\&J and AZN vaccines have safety concerns regarding blood clotting. For the AZN vaccine, European regulators announced on March 7th that they had found possible links between the vaccine and very rare cases of blood clots. On the same day, EMA's safety committee (PRAC) suggested unusual blood clots with low blood platelets as new rare side effects [18]. Reuters also recommended that AZN's COVID-19 shot only be used on those over 60 due to this link [19]. The J\&J vaccine also has similar concerns and was called for a pause earlier April 2021 by the CDC and the U.S. Food and Drug Administration (FDA) after receiving a small number of reports of a rare and severe type of blood clot happening in people who got this vaccine [20]. The J\&J vaccine is only authorized for people older than 18 years old. And very little data are available to assess pregnancy vaccine safety for both these vaccines [21]. 


\section{MRNA Vaccine}

\subsection{Mechanism}

Moderna and Pfizer/BioNTech vaccines are made using mRNA, a technology that can deliver genetic code to cells. This technology makes a spike in the SARS-2 virus. The immune system is activated by the proteins made with the mRNA instructions, recognizes the spike protein as a foreign signal, and develops antibodies and other immunity weapons to fight against the similar virus in the future [22].

\subsection{Efficacy and Effectiveness}

For mRNA vaccine, an effectiveness rate of around $95 \%$ has been observed. For example, the Moderna vaccine is 94\% effective, and Pfizer says its vaccine was 95\% effective in preventing COVID-19 cases with symptoms [23]. Both of them are $100 \%$ effective when it came to preventing severe cases [23] as illustrated in [23, Fig. 1]. Moreover, they have both also been shown to provide immunogenicity for at least 119 days after the first vaccination [24] as presented in [24, Fig. 2]. In terms of efficacy to different people, the Moderna vaccine's efficacy is lower in people 65 years old and older; however, the company said this result might because the trial did not have many of that age group's cases. For different ethnic and racial groups, the vaccine is equally effective [22]. For Pfizer/BioNTech vaccine, vaccine efficacy was similar across subgroups defined by age, sex, race, ethnicity, baseline body-mass index, and the presence of coexisting conditions [25].

\subsection{Side Effects}

Moderna vaccine and Pfizer/BioNTech vaccine share similar side effects such as pain, swelling at the injection site, fatigue, and headache. As for severe side effects, the Moderna vaccine is a bit higher than Pfizer/BioNTech vaccine. Moderna's severe side effects included fatigue in $9.7 \%$ of participants, muscle pain in $8.9 \%$, joint pain in $5.2 \%$, and headache in $4.5 \%$, while for Pfizer/BioNTech vaccine trial, only $3.8 \%$ experience fatigue, $2 \%$ for headache [26].

\section{Comparison}

\subsection{Concerns for all Countries}

\subsubsection{Effectiveness}

If countries or patients only consider effectiveness, Moderna and Pfizer/BioNTech vaccines might win the game since they have the highest effectiveness rate, around $95 \%$ for symptomatic Covid-19. But if only effective against severe disease is considered, AstraZeneca COVID-19 vaccine, Moderna vaccine, and
Pfizer/BioNTech vaccine are the same as they are $100 \%$ effective.

Even though the effectiveness is about the same across all vaccine types, there is a considerable variation in their clinical trial size. Inactivated vaccines (CoronaVac) have a clinical trial involving 1322 participants; viral vector vaccines (Oxford-AstraZeneca COVID-19 vaccine) have 23848 participants enrolled [27]; mRNA has a trial of 30420 volunteers [28]. Therefore, mRNA vaccines might have higher reliability.

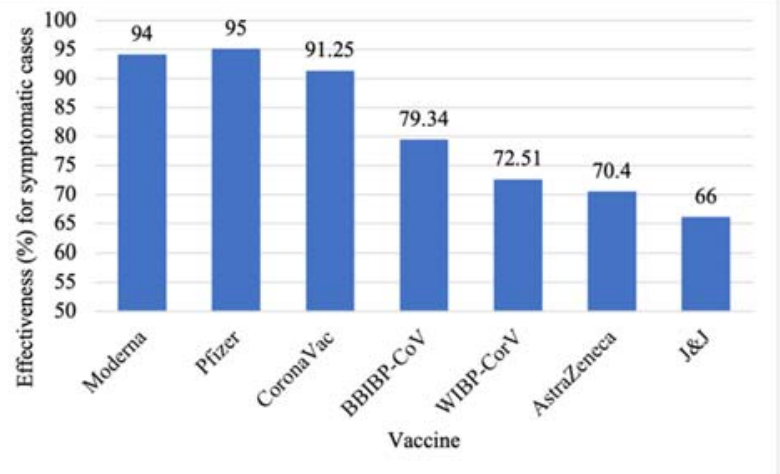

Figure 1. Effectiveness of Various COVID -19 Vaccines. Data in the figure adapted from $[3][4][5][10][11][12][23]$

\subsubsection{Cost performance ratio}

Since one way to fully elevate a country from the impact of this pandemic is probably vaccine, the vaccine may need to be taken by nearly all people, leading to a great financial burden to the country. When considering this, the AstraZeneca COVID-19 vaccine may have the greatest advantage as an inoculator only pays 3 dollars per dose. It is cheap compared to $\$ 32-37$ Moderna vaccine, \$19.05 Pfizer/BioNTech, \$10 Johnson \& Johnson's COVID-19 vaccine, and \$29.75 CoronaVac per dose [24] [29][30]. For more details, the reader is referred to [29, 30, Fig. 2]

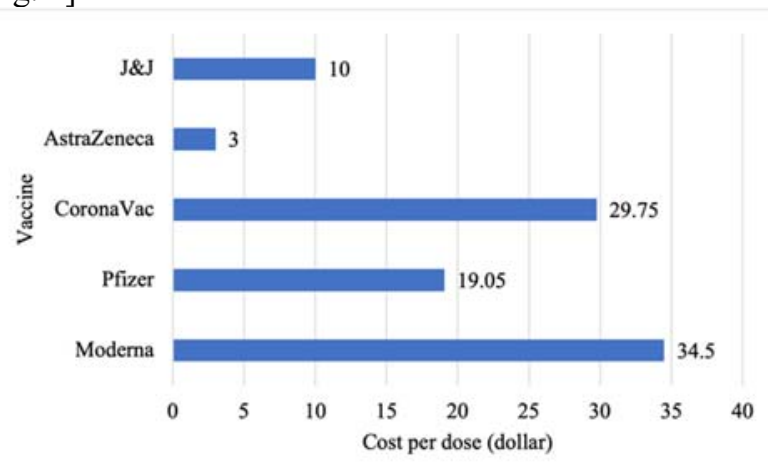

Figure 2. Cost of Various COVID -19 Vaccines. Data in the figure adapted from [24][29][30]

Besides the cost, mass production is another important factor of the vaccine's cost-effectiveness. CoronaVac and AstraZeneca vaccines are the best choice with an annual capacity of 2 billion doses based on presently available data. While for Moderna increased its global production estimate to 600 million doses for 2021 and potentially up to 1 billion [31]. Pfizer expects to produce up to 1.3 billion doses in 2021 [32]. Sinovac had started to double CoronaVac's annual capacity to 2 billion doses [33]. 
AstraZeneca has doubled the manufacturing capacity for its potential Covid-19 vaccine to 2 billion doses [34].

Convenient transportation condition is also very important for putting into mass use. For transportation, viral-based vector vaccines are more stable than mRNA vaccines. For mRNA vaccines, the Moderna vaccine, compared to Pfizer/BioNTech vaccine, is easier to transport and store because it is less temperature sensitive [24].

\subsection{Concerns Specific to Regions}

\subsubsection{Age}

As the aging population becomes an issue in many countries, especially the developed ones, the effects and side-effects of the vaccines to the elderly should be taken into consideration. The Pfizer/BioNTech and Moderna vaccines are still unclear that the efficacy is relatively low for people aged above 65 . The effectiveness and safety of the vaccine in the elderly are proved in CoronaVac. Thus, it is suggested that many developed countries with aging populations can choose to adopt vaccines safe and effective for the old, such as CoronaVac. As seen in [35,36,37, Tab.1], we can see the recommended ages for receiving the five commonly used vaccines: people 18 years and older are suitable for receiving Moderna, J\&J and SinoVac doses[35]; people 12 years and older are recommended for Pfizer vaccination[36]; the recommended age for receiving Astrazeneca vaccine is not yet available by test[37].

Table1. Age Recommended for Various COVID -19

Vaccines. Data in the table adapted from [35][36][37]

\begin{tabular}{|c|c|}
\hline Vaccine & Who is it recommended for(in age)? \\
\hline Pfizer & People 12 years and older. \\
\hline Moderna & People 18 years and older. \\
\hline Astrazeneca & Not yet available. \\
\hline Johnson \& Johnson & People 18 years and older. \\
\hline Sinovac & People 18 years and older. \\
\hline
\end{tabular}

\subsubsection{Race}

The effectiveness of the Pfizer/BioNTech Vaccine is proved across human races. Besides, the clinical trial of CoronaVac is conducted in many countries in South American, Asia, North America, and Europe. No data supporting race-related side effects are collected.

\subsubsection{Mutant Variants Distribution}

As mentioned above, the current variants mainly include B.1.1.7, B.1.351, and P.1. The B.1.1.7 variants are spreading in Asia and Europe; B.1.351 and P.1 in Europe and South America.[38] Since the SARS-CoV-2 virus is constantly mutating, and the distribution of the mutants are not even on the world scale, it is necessary to identify the vaccine effective to the variance popular in the country. CoronaVac was efficient against three variants of COVID19 worldwide, including British B.1.1.7, South African 501.V2, and Brazil's P.1. [39]. Since CoronaVac and other inactivated virus vaccines have all parts of the virus, the type of vaccine will induce a sufficient amount of immune response and is less susceptible to viral mutations. The effectiveness of various vaccines against major variants of SARS-CoV-2 virus are shown in table [40, Tab.2].

Table2. Variant Effectiveness for Various COVID-19 Vaccines. Data in the table adapted from [40]

\begin{tabular}{|c|c|}
\hline Vaccine & Effectiveness against Variants \\
\hline Pfizer & $\begin{array}{c}\text { Shown to be "quite effective" against the } \\
\text { UK variant as well as the South African and } \\
\text { Latin American variant. }\end{array}$ \\
\hline Moderna & $\begin{array}{c}\text { Shown to be "quite effective" against the } \\
\text { UK variant as well as the South African and } \\
\text { Latin American variants. }\end{array}$ \\
\hline Astrazeneca & $\begin{array}{c}\text { At least one study finds it has little effect } \\
\text { against the South African variant, but } \\
\text { appears effective against UK and Brazilian } \\
\text { variants. }\end{array}$ \\
\hline Johnson \& \\
Johnson & $\begin{array}{c}\text { Based on clinical studies in Africa, UK and } \\
\text { Latin America, there is evidence the } \\
\text { vaccine is effective against the variants, } \\
\text { although less so against the South African } \\
\text { and Latin American strains. }\end{array}$ \\
\hline Sinovac & $\begin{array}{c}\text { Unknown, although a study in Brazil } \\
\text { demonstrated 50.4\% efficacy at preventing } \\
\text { symptomatic infections. }\end{array}$ \\
\hline
\end{tabular}

\subsubsection{The Maturity of Technique}

Many countries are most concerned about how mature the techniques that could be used for vaccine development are, which may also be associated with safety concerns. We conclude that using an inactivated vaccine is a safer way of doing vaccination [41]. mRNA vaccines have reported reactogenicity (the physical manifestation of the inflammatory response to vaccination) [42] but are not considered as something adverse enough. And viral-based vaccines have raised considerable concerns with their link to blood clot formation [19][42]. In comparison, inactivated vaccines use the most conservative technique and show the most moderate side effects and few cases of adverse effects so far.

\subsubsection{Time for Developing New Vaccine}

After considering safety issues in priority, ensuring the effectiveness of vaccination against all types of variants is another crucial concern for all countries. Since Virus is notorious due to its rapid mutation, the time needed to 
develop new vaccines adaptive to those variants matters. Based on our findings [43], we conclude that developing a new mRNA vaccine takes the least amount of time. This is because it is very easy to synthesize mRNA that encodes a viral protein once researchers know the targeting viral sequence. For the other two types of traditional vaccines, researchers need to take a long time modifying the infectious viruses, and producers need to input a lot of resources to manufacture the protein or the virus [44].

\section{Conclusion}

This paper reviews the latest studies regarding COVID19 vaccines of the three most popular types, including the inactivated virus vaccines, the viral vector vaccines, and the mRNA vaccines. Although every vaccine has its drawbacks and merits, suggestions are made to choose vaccines based on many factors. As for the developed countries with a relatively large proportion of the aged population, it is safer to apply the inactivated virus vaccine. However, for the nations and territories facing the rapid mutating SARS-CoV-2, mRNA vaccines are a better choice due to their effectiveness in deterring the variants. The effectiveness of the vaccines in distinct races remained unclear. Considering the efficacy, the Moderna and Pfizer/BioNTech vaccines own the highest effectiveness and reliability. In terms of the cost for mass production and price for injection, the CoronaVac and AstraZeneca vaccines are the cheapest vaccines for manufacturing on the market. The AstraZeneca COVID19 vaccine has the lowest price for individuals. Since people may trust the vaccines researched and developed in their own countries, it would be helpful for popular acceptance to apply the nation's self-developed vaccines.

\section{References}

1. "Coronavirus disease 2019 (COVID-19)," Mayo Clinic, 15-Apr-2021. [Online]. Available: https://www.mayoclinic.org/diseasesconditions/coronavirus/symptoms-causes/syc20479963. [Accessed: 27-Apr-2021].

2. F. Li, "Receptor Recognition Mechanisms of Coronaviruses: a Decade of Structural Studies," Journal of Virology, vol. 89, no. 4, pp. 1954-1964, 2014.

3. S. Mallapaty, "China COVID vaccine reports mixed results - what does that mean for the pandemic?," Nature, 2021, doi: 10.1038/d41586-021-00094-z.

4. R. Staff, "China approves two more domestic COVID-19 vaccines for public use," Reuters, 2021.

5. S.-L. Wee, "China approves two more Covid-19 vaccines," The New York Times, 2021.

6. Z. $\mathrm{Wu}$ et al., "Safety, tolerability, and immunogenicity of an inactivated SARS-CoV-2 vaccine (CoronaVac) in healthy adults aged 60 years and older: a randomised, double-blind, placebocontrolled, phase $1 / 2$ clinical trial," The Lancet
Infectious Diseases, 2021, doi: 10.1016/S14733099(20)30987-7.

7. S. Xia et al., "Safety and immunogenicity of an inactivated SARS-CoV-2 vaccine, BBIBP-CorV: a randomized, double-blind, placebo-controlled, phase 1/2 trial," The Lancet Infectious Diseases, vol. 21, no. 1, pp. 39-51, Jan. 2021, doi: 10.1016/S14733099(20)30831-8.

8. J. Huang, H. Huang, D. Wang, C. Wang, and Y. Wang, "Immunological strategies against spike protein: Neutralizing antibodies and vaccine development for COVID-19," Clinical and Translational Medicine, vol. 10, no. 6, Oct. 2020, doi: 10.1002/ctm2.184.

9. "Understanding Viral Vector COVID-19 Vaccines," Centers for Disease Control and Prevention. [Online]. Available: https://www.cdc.gov/coronavirus/2019ncov/vaccines/different-vaccines/viralvector.html. [Accessed: 27-Apr-2021].

10. M. Voysey and A. J. Pollard, "ChAdOx1 nCoV-19 vaccine for SARS-CoV-2 - Authors' reply," The Lancet, vol. 396, no. 10261, pp. 1486-1487, 2020.

11. M. D. Knoll and C. Wonodi, "Oxford-AstraZeneca COVID-19 vaccine efficacy," The Lancet, vol. 397, no. 10269, pp. 72-74, 2021.

12. "Phase III Double-blind, Placebo-controlled Study of AZD1222 for the Prevention of COVID-19 in Adults - Full Text View," Full Text View ClinicalTrials.gov. [Online]. Available: https:/clinicaltrials.gov/ct2/show/NCT04516746. [Accessed: 27-Apr-2021].

13. S. Owermohle, "FDA confirms J\&J vaccine is safe and effective," POLITICO, 24-Feb-2021. [Online]. Available:

https:/www.politico.com/news/2021/02/24/fdajohnson-johnson-single-dose-shot-covid-471327. [Accessed: 27-Apr-2021].

14. A. Koop, "Tracking COVID-19 Vaccines Around the World," Visual Capitalist, 25-Jan-2021. [Online]. Available:

https://www.visualcapitalist.com/tracking-covid-19vaccines-around-the-world/. [Accessed: 27-Apr$2021]$.

15. S. Ebbs, "Single-dose Johnson \& Johnson vaccine effective against COVID-19 variants: Data," ABC7 New York, 24-Feb-2021. [Online]. Available: https://abc7ny.com/single-dose-johnson-andjohnson-vaccine-effective-against-covid-19variants-data/10366833/. [Accessed: 27-Apr-2021].

16. “Johnson \& Johnson," Content Lab U.S.[Online]. Available: https://www.jnj.com/johnson-johnsoncovid-19-vaccine-authorized-by-u-s-fda-foremergency-usefirst-single-shot-vaccine-in-fightagainst-global-pandemic. [Accessed: 27-Apr-2021].

17. 2021 Lianna Matt McLernon | News Writer | CIDRAP News | Mar 31, "AstraZeneca COVID vaccine $70 \%$ effective vs B117 variant," CIDRAP, 31-Mar-2021. [Online]. Available: https://www.cidrap.umn.edu/news- 
perspective/2021/03/astrazeneca-covid-vaccine-70effective-vs-b117-variant. [Accessed: 27-Apr-2021].

18. A. C. PINHO, “AstraZeneca's COVID-19 vaccine: EMA finds possible link to very rare cases of unusual blood clots with low platelets," European Medicines Agency, 08-Apr-2021. [Online]. Available: https:/www.ema.europa.eu/en/news/astrazenecascovid-19-vaccine-ema-finds-possible-link-very-rarecases-unusual-blood-clots-low-blood. [Accessed: 27-Apr-2021].

19. K. Kelland, G. Jones, and F. Guarascio, "Italy, Britain suggest age limits for AstraZeneca vaccine but still recommend it," Reuters, 07-Apr-2021. [Online]. Available: https://www.reuters.com/article/us-healthcoronavirus-astrazeneca-idUSKBN2BU27C. [Accessed: 27-Apr-2021].

20. S. Owermohle, "FDA confirms J\&J vaccine is safe and effective," POLITICO, 24-Feb-2021. [Online]. Available:

https://www.politico.com/news/2021/02/24/fdajohnson-johnson-single-dose-shot-covid-471327. [Accessed: 27-Apr-2021].

21. "Covid: How does the Oxford-AstraZeneca vaccine work?," BBC News, 15-Apr-2021. [Online]. Available: https://www.bbc.com/news/health55302595. [Accessed: 27-Apr-2021].

22. H. Branswell, "Comparing the Covid-19 vaccines developed by Pfizer, Moderna, and Johnson \& Johnson," STAT+, 2 February 2021. [Online]. Available:

https:/www.statnews.com/2021/02/02/comparingthe-covid-19-vaccines-developed-by-pfizermoderna-and-johnson-johnson/. [Accessed 12 April 2021].

23. A. Martichoux, "AstraZeneca, Pfizer, Moderna, Johnson \& Johnson: Breaking down the differences between coronavirus vaccines," abc7news, 25 March 2021. [Online]. Available: https://abc7news.com/best-covid-vaccineastrazeneca-moderna-most-effective/10441395/. [Accessed 12 April 2021].

24. I. A. B. J. A. A. S. M. D. C. K. S A Meo, "COVID-19 vaccines: comparison of biological, pharmacological characteristics and adverse effects of Pfizer/BioNTech and Moderna Vaccines," PubMed.gov, 2021.

25. S. J. T. ,. N. K. ,. J. A. ,. A. G. ,. S. L. ,. J. L. P. ,. G. P. M. ,. E. D. M. ,. C. Z. ,. R. B. ,. K. A. S. ,. S. R.,. K. Fernando P Polack, "Safety and Efficacy of the BNT162b2 mRNA Covid-19 Vaccine," The new England journal of medicine, 2020.

26. M. Wadman, "Public needs to prep for vaccine side effects," Science, vol. 370, no. 6520, 2020.

27. R. Staff, "Fact check: COVID-19 vaccines did have clinical trials," Reuters, 3 February 2021. [Online]. Available: $\quad$ https://www.reuters.com/article/ukfactcheck-vaccines-clinical-trials-idUSKBN2A22D3. [Accessed 12 April 2021].
28. M. H. M. E. S. M. B. E. M. K. K. M. S. F. M. R. N. M. D. D. M. S. A. S. M. N. R. M. C. B. C. M. J. M. M. K. Lindsey R. Baden, "Efficacy and Safety of the mRNA-1273 SARS-CoV-2 Vaccine," The New England journal of Medicine, 2021.

29. S. Cao, "COVID-19 Vaccine Prices Revealed From Pfizer, Moderna, and AstraZeneca," 23 November 2020. [Online]. Available: https://observer.com/2020/11/covid19-vaccineprice-pfizer-moderna-astrazeneca-oxford/. [Accessed 29 April 2021].

30. R. Staff, "Sinovac coronavirus vaccine offered by Chinese city for emergency use costs $\$ 60$," reuters, 16 October 2020. [Online]. Available: https://www.reuters.com/article/us-healthcoronavirus-china-vaccine-idUSKBN2710UQ. [Accessed 29 April 2021].

31. "Moderna Provides COVID-19 Vaccine Supply Update," Moderna, 4 January 2021. [Online]. Available: https://investors.modernatx.com/newsreleases/news-release-details/moderna-providescovid-19-vaccine-supply-update. [Accessed 12 April $2021]$.

32. "MANUFACTURING AND DISTRIBUTING THE COVID-19 VACCINE," Pfizer, [Online]. Available: https://www.pfizer.com/science/coronavirus/vaccine /manufacturing-and-distribution. [Accessed 12 April 2021].

33. R. Staff, "China Sinovac says it reached two billion doses annual capacity for COVID-19 vaccine," Reuters, 2 April 2021. [Online]. Available: https://www.reuters.com/article/us-healthcoronavirus-vaccine-sinovac-idUSKBN2BP07G. [Accessed 12 April 2021].

34. k. Makortoff, "AstraZeneca doubles capacity for potential Covid-19 vaccine to $2 \mathrm{bn}$ doses," The guardian, 4 June 2020. [Online]. Available: https://www.theguardian.com/business/2020/jun/04/ astrazeneca-doubles-capacity-for-potential-covid19-vaccine-to-2bn-doses. [Accessed 12 April 2021].

35. Centers for Disease Control and Prevention, "Coronavirus Disease 2019 (COVID-19)," Centers for Disease Control and Prevention, Feb. 11, 2020. https://www.cdc.gov/coronavirus/2019ncov/vaccines/different-vaccines.html.

36. "The Sinovac COVID-19 vaccine: What you need to know," www.who.int. https://www.who.int/newsroom/feature-stories/detail/the-sinovac-covid-19vaccine-what-you-need-to-know.

37. W. N. Staff, "Three COVID Vaccines Compared," WebMD. https://www.webmd.com/vaccines/covid19-vaccine/news/20201214/closer-look-at-threecovid-19-vaccines.

38. E. Mahase, "Covid-19: Where are we on vaccines and variants?," BMJ (Clinical research ed.), vol. 372, p. n597, Mar. 2021, doi: 10.1136/bmj.n597.

39. N. Franco, "Estudos mostram eficácia da CoronaVac contra três variantes do vírus," Repórter da Agência Brasil, 2021. 
40. M. Terry, "Comparing COVID-19 Vaccines: Timelines, Types and Prices," BioSpace, Feb. 08, 2021. https://www.biospace.com/article/comparingcovid-19-vaccines-pfizer-biontech-modernaastrazeneca-oxford-j-and-j-russia-s-sputnik-v/.

41. C. N. N. P. Staff, "Inactivated vaccine safer way of doing vaccination, expert says," cnn. [Online]. Available:

https://www.cnnphilippines.com/news/2021/1/12/ina ctivate-vaccine-coronavirus-safety.html. [Accessed: 27-Apr-2021].

42. S. Cao, "COVID-19 Vaccine Prices Revealed From Pfizer, Moderna, and AstraZeneca," Observer, 23Nov-2020. [Online]. Available: https://observer.com/2020/11/covid19-vaccineprice-pfizer-moderna-astrazeneca-oxford/. [Accessed: 27-Apr-2021].

43. "COVID-19 Vaccine FAQs," ASM.org. [Online]. Available: https://asm.org/Articles/2020/December/COVID19-Vaccine-FAQs. [Accessed: 27-Apr-2021].

44. Anne Trafton|MIT News Office, "Explained: Why RNA vaccines for Covid-19 raced to the front of the pack," MIT News | Massachusetts Institute of Technology. [Online]. Available: https://news.mit.edu/2020/rna-vaccines-explainedcovid-19-1211. [Accessed: 27-Apr-2021]. 